\title{
A Retrospective Analysis for Patients with HER2-Positive Gastric Cancer Who Were Treated with Trastuzumab-Based Chemotherapy: In the Perspectives of Ethnicity and Histology
}

\author{
Jun Ho Yi, MD, PhD' \\ Jung Hun Kang, MD, $P h D^{2}$ \\ In Gyu Hwang, MD, PhD \\ Hee Kyung Ahn, MD, PhD ${ }^{4}$ \\ Hyun Jin Baek, MD \\ Soon II Lee, MD, PhD \\ Do Hyoung Lim, MD, PhD 5 \\ Young-Woong Won, $\mathrm{MD}, \mathrm{PhD}^{6}$ \\ Jun $\mathrm{Ho}$ Ji, MD, PhD? \\ Hyo Song Kim, MD, PhD ${ }^{8}$ \\ Sun Young Rha, MD, PhD \\ Sung Yong $\mathbf{O h}, \mathrm{MD}, \mathrm{PhD}^{9}$ \\ Kyung Eun Lee, MD, PhD ${ }^{10}$ \\ Taekyu Lim, MD, PhD'11 \\ Chi Hoon Maeng, MD, PhD'2 \\ Moon Jin Kim, MD ${ }^{13}$ \\ Seung Tae Kim, MD, PhD ${ }^{13}$ \\ Jeeyun Lee, MD, $\mathrm{PhD}^{13}$ \\ Joon Oh Park, MD, PhD ${ }^{13}$ \\ Young Suk Park, MD, PhD ${ }^{13}$ \\ Ho Yeong Lim, MD, PhD ${ }^{13}$ \\ Won Ki Kang, MD, PhD ${ }^{13}$ \\ Se Hoon Park, MD, PhD ${ }^{13}$
}

* A list of authors' affiliations appears at the end of the paper.

\section{Purpose}

While the Trastuzumab for Gastric Cancer (ToGA) trial demonstrated the efficacy and safety of trastuzumab-based chemotherapy in HER2-positive metastatic gastric cancer, the overall survival (OS) benefit was not found in Asian and diffuse-type cancer patients. The aim of the study is to investigate predictive markers for trastuzumab-based chemotherapy.

\section{Materials and Methods}

Data of patients with HER2-positive gastric cancer treated with trastuzumab-based chemotherapy were analyzed retrospectively.

\section{Results}

A total of 168 Asian patients were included. The median age was 60 years (range, 27 to 85 years) and the male:female ratio was 118 (70.2\%):50 (29.8\%). Fourteen (8.3\%), 63 (37.5\%), 75 (44.6\%), and 11 (6.5\%) patients had well, moderately, poorly-differentiated tubular adenocarcinoma and signet ring cell carcinoma, respectively. With 14 complete responses and 73 partial responses, the response rate was $50.6 \%$. The median progression-free survival (PFS) was 10.2 months (95\% confidence interval [Cl], 8.7 to 11.7), and the median OS was 18.5 months ( $95 \% \mathrm{Cl}, 16.4$ to 50.6). Next, we investigated the effect of poorly-differentiated histology (PDH, poorly-differentiated tubular adenocarcinoma+signet ring cell carcinoma) on clinical outcomes. The median PFS (8.9 months vs. 11.5 months, $p=0.16$ ) was slightly inferior in $\mathrm{PDH}$ patients, and the median OS was significantly shorter in PDH patients (14.6 months vs. 19.0 months, $p=0.025)$.

\section{Conclusion}

While subset analysis of the ToGA trial demonstrated that trastuzumab-based chemotherapy may not be beneficial for Asians and patients with PDH, our data may suggest that even in Asian patients and patients with PDH, trastuzumab-based chemotherapy could be associated with improved clinical outcomes in patients with HER2-positive gastric cancer.

\section{Key words}

Stomach neoplasms, ErbB-2 receptor, Trastuzumab, Histology, Ethnic groups
Correspondence: Se Hoon Park, MD, PhD

Division of Hematology-Oncology,

Department of Medicine,

Samsung Medical Center,

Sungkyunkwan University School of Medicine, 81 Irwon-ro, Gangnam-gu, Seoul 06351, Korea Tel: 82-2-3410-1767

Fax: 82-2-3410-1754

E-mail: hematoma@skku.edu

Received May 8, 2015

Accepted July 13, 2015

Published Online August 10, 2015 


\section{Introduction}

Gastric cancer is the fifth most common cancer worldwide, with an estimated occurrence of almost one million new cases every year, with half of the world total occurring in East Asia [1]. In terms of mortality, it remains one of the most common causes of cancer related mortality, only second to lung cancer [2], as less than $50 \%$ of patients are eligible for complete resection, and, in spite of recent progress, expected survival duration with palliative treatment barely exceeds 1 year $[3,4]$.

HER2 amplification or overexpression is known to occur in approximately $15 \%-20 \%$ of patients with gastric cancer. The pivotal Trastuzumab for Gastric Cancer (ToGA) trial [5] demonstrated that patients with HER2-positive gastric or gastroesophageal junction (GEJ) cancer may benefit from trastuzumab, an anti-HER2 monoclonal antibody. In the ToGA trial, patients assigned to receive trastuzumab in combination with chemotherapy showed significant improvement in overall survival (OS, 13.8 vs. 11.1 months), progression-free survival (PFS, 6.7 months vs. 5.5 months), and overall response rate (ORR, $47 \%$ vs. $35 \%$ ). In addition, an exploratory analysis demonstrated that patients with strong protein expression (immunohistochemistry [IHC] $3[+]$ or IHC 2[+]/ fluorescence in situ hybridization [FISH] [+]) were more likely to have favorable OS with the addition of trastuzumab (16.0 months vs. 11.8 months). With these results, trastuzumab was approved as a standard treatment option for HER2-positive gastric cancer patients.

Meanwhile, the subset analysis of the ToGA trial generated several questions. It is well-known that ethnicity matters in gastric cancer [6]. Not only in terms of epidemiology, histology, or pharmacodynamics, certain treatments which were effective for a Western population did not show similar efficacy in an Asian population, as in the AVAGAST trial [7]. In fact, in the subset analysis of the ToGA trial, OS benefit was not observed in the Asian population, while patients from America and Europe did benefit from the treatment. In addition, according to the subset analysis of the ToGA trial, patients with diffuse-type cancer who seldom harbor HER2 overexpression did not show OS benefit from trastuzumabbased treatment.

In the current study, we conducted a multicenter, retrospective analysis on HER2-positive gastric cancer patients from 12 tertiary institutes in Korea, who were treated with trastuzumab-based treatment in order to confirm whether the clinical outcomes are affected by Asian ethnicity or histology, and to provide further understanding of the disease nature of HER2-positive gastric cancer.

\section{Materials and Methods}

\section{Patients}

Patients diagnosed with HER2-positive unresectable or metastatic gastric cancer from 2009 to 2014 were included in the analysis. The inclusion criteria were as follows: (1) a pathologically confirmed diagnosis of gastric cancer; (2) HER2 positivity confirmed according to the criteria used in the ToGA trial, that is, either IHC 3(+) or IHC 2(+)/ FISH (+); (3) treated with trasutuzumab-based chemotherapy as the firstline treatment. Combined therapy could be continued after six cycles according to physicians' decision. (4) Complete medical records, including demographics, site of primary tumor, and pathologic reports. The study was approved by the Institutional Review Board of the primary investigator's institute, Samsung Medical Center.

\section{Clinicopathologic parameters}

Clinicopathologic parameters including age, sex, performance status, primary tumor site, Lauren's classification, World Health Organization (WHO) histologic classification, measurability of lesions, methods for HER2 confirmation, backbone chemotherapeutic regimen, and baseline laboratory findings were collected retrospectively from patients' medical records.

\section{Statistical analysis}

OS was measured from the date of diagnosis of an unresectable or metastatic disease to the date of death or last follow-up, and PFS was measured from the date of diagnosis of a metastatic or unresectable disease to the date of progressive disease, death, or last follow-up. All survival parameters were calculated using the Kaplan-Meier method and were compared using a log-rank test. Tumor response was assessed using Response Evaluation Criteria in Solid Tumors ver. 1.0. p-values less than 0.05 were considered statistically significant, and all p-values corresponded to two-sided significance tests.

To assess whether higher HER2 / CEP17 ratio affected PFS, patients were dichotomized according to good survival group (median PFS $\geq 6$ months) versus poor survival group (median PFS $<6$ months). The optimal cutoff for HER2/ CEP17 ratio for predicting a favorable PFS was determined using receiver operating characteristic (ROC) curve analyses, and further evaluation was performed using the area under the curve (AUC). All statistical analyses were performed using SPSS ver. 17 (SPSS Inc., Chicago, IL). 


\section{Results}

\section{Demographics and baseline disease characteristics}

A total of 168 patients from 12 institutes were eligible for the analysis. The median age of patients was 60 years (range, 27 to 85 years) and 118 patients $(70.2 \%)$ were male. All patients were Asian. Regarding the location of the primary tumor, 161 cases $(95.8 \%)$ were stomach cancer and seven cases $(4.2 \%)$ were GEJ cancer. As many pathologists in Korea do not interpret Lauren's classification with biopsied specimens, only 78 cases had been confirmed using Lauren's classification, and among them, $44(56.4 \%), 22(28.2 \%)$, and 12 cases $(15.4 \%)$ were found to be intestinal-type, diffuse-type, and mixed-type cancer, respectively. However, WHO histologic classification was confirmed in all cases, with $14(8.3 \%)$, $63(37.5 \%), 75(44.6 \%)$, and 11 cases $(6.5 \%)$ found to be welldifferentiated, moderately-differentiated, poorly-differentiated tubular adenocarcinoma, and signet ring cell carcinoma, respectively. In terms of methods for HER2 confirmation, in 129 patients disease was confirmed by IHC 3(+), and by IHC 2-3(+)/FISH (+) in 59 patients whose median value of HER2/CEP17 ratio was 3.34 (range, 2.09 to 20.00). Other details are described in Table 1.

\section{Clinical outcomes of trastuzumab-based therapy}

Regarding the backbone regimen of the trastuzumabbased therapy, 145 patients $(86.3 \%)$ had received capecitabine plus cisplatin, 14 patients $(8.3 \%)$ had received 5 - fluorouracil (5-FU) plus cispaltin, and nine patients had received other agents. Median number of cycles was six (range, 1 to 62) for both trastuzumab and chemotherapy. Tumor responses were as follows: $12(7.1 \%)$ complete response, $73(43.5 \%)$ partial response, $48(28.6 \%)$ stable disease, $17(10.1 \%)$ progressive disease, and $18(10.7 \%)$ cases were not evaluable, resulting in $50.6 \%$ of ORR (Table 2 ).

With a median follow-up duration of 32.9 months (95\% confidential interval [CI], 30.0 to 35.8), median PFS was 10.2 months (95\% CI, 8.7 to 11.7), and median OS was 18.5 months (95\% CI, 16.4 to 50.6). Considering that Asian patients usually achieve approximately 12 months of OS and 6 months of PFS, the numerical data of the current study may suggest that even in an Asian population, trastuzumab-based therapy could be beneficial for patients with HER2-positive gastric cancer.

Next, subset analysis was performed for survival according to the histologic differentiation. Patients with well-differentiated and moderately-differentiated tubular adenocarcinoma were categorized as the well-differentiated histologic (WDH) group, and poorly-differentiated tubular adenocar-
Table 1. Demographics and baseline disease characteristics

\begin{tabular}{|c|c|}
\hline Characteristic & o. $(\%)(n=168)$ \\
\hline Age, median (range, yr) & $60(27-85)$ \\
\hline \multicolumn{2}{|l|}{ Sex } \\
\hline Male & $118(70.2)$ \\
\hline Female & $50(29.8)$ \\
\hline \multicolumn{2}{|l|}{ ECOG performance status } \\
\hline 0 & $45(26.8)$ \\
\hline 1 & $115(68.5)$ \\
\hline 2 & $7(4.2)$ \\
\hline 3 & $1(0.6)$ \\
\hline \multicolumn{2}{|l|}{ Primary tumor site } \\
\hline Gastroesophageal junction & $7(4.2)$ \\
\hline Stomach & $161(95.8)$ \\
\hline \multicolumn{2}{|l|}{ Previous gastrectomy } \\
\hline Not done & $99(58.9)$ \\
\hline Subtotal gastrectomy & $45(26.8)$ \\
\hline Total gastrectomy & $24(14.3)$ \\
\hline \multicolumn{2}{|l|}{ Extent of disease } \\
\hline Locally advanced & $54(32.1)$ \\
\hline Metastatic & $114(67.9)$ \\
\hline \multicolumn{2}{|l|}{ Disease measurability } \\
\hline Measurable & $97(57.7)$ \\
\hline Non-measurable & $71(42.3)$ \\
\hline \multicolumn{2}{|l|}{ HER2 status } \\
\hline IHC 3(+)/FISH not done & 109 \\
\hline IHC 2(+)/FISH (+) & 39 \\
\hline $\operatorname{IHC} 3(+) /$ FISH $(+)^{a)}$ & 20 \\
\hline \multicolumn{2}{|l|}{ WHO histologic classification } \\
\hline Well-differentiated tubular adenocarcinoma & $14(8.3)$ \\
\hline $\begin{array}{l}\text { Moderately-differentiated tubular } \\
\text { adenocarcinoma }\end{array}$ & $63(37.5)$ \\
\hline Poorly-differentiated tubular adenocarcinoma & $75(44.6)$ \\
\hline Signet ring cell carcinoma & $11(6.5)$ \\
\hline Others & $5(3.0)$ \\
\hline \multicolumn{2}{|l|}{ Lauren's classification $(\mathrm{n}=78)$} \\
\hline Intestinal & $44(56.4)$ \\
\hline Diffuse & $22(28.2)$ \\
\hline Mixed & $12(15.4)$ \\
\hline \multicolumn{2}{|l|}{ Specimens confirmed as HER2 $(+)^{\text {b) }}$} \\
\hline Surgical specimen & 61 \\
\hline Endoscopic biopsied specimen & 108 \\
\hline Specimens from metastatic site & 12 \\
\hline
\end{tabular}

ECOG, Eastern Cooperative Oncology Group; IHC, immunohistochemistry; FISH, fluorescence in situ hybridization; WHO, World Health Organization. ${ }^{a}$ Both IHC and FISH were performed in these patients, b)Multiple specimens could be sampled in a single patient. 
Table 2. Clinical outcomes of trastuzumab-based treatment

\begin{tabular}{|lc}
\hline Variable & No. (\%) \\
\hline Backbone regimen & \\
\hline XP & $145(86.3)$ \\
\hline FP & $14(8.3)$ \\
XELOX & $6(3.6)$ \\
\hline Capecitabine & $2(1.2)$ \\
\hline None (trastuzumab monotherapy) & $1(0.6)$ \\
\hline Tumor response & \\
\hline Complete response & $12(7.1)$ \\
\hline Partial response & $73(43.5)$ \\
\hline Stable disease & $48(28.6)$ \\
\hline Progressive disease & $17(10.1)$ \\
\hline Missing & $18(10.7)$ \\
\hline Overall survival, median $\mathbf{( 9 5 \% ~ C I , ~ m o ) ~}$ & $18.5(16.4-50.6)$ \\
\hline Progression-free survival, & $10.2(8.8-11.6)$ \\
median (95\% CI, mo) & \\
\hline
\end{tabular}

$\mathrm{XP}$, capecitabine plus cisplatin; FP, 5-fluorouracil (5-FU) plus cisplatin; XELOX, capecitabine and oxaliplatin; CI, confidence interval.

cinoma and signet ring cell carcinoma as poorly-differentiated histologic (PDH) group. Consequently, $77(45.8 \%)$ and $86(51.2 \%)$ patients were classified as the WDH and PDH group, respectively. The median PFS of the WDH group was slightly longer than that of the PDH group, which did not reach statistical significance (11.5 months [95\% CI, 8.1 to 14.9] vs. 8.9 months [95\% CI, 7.2 to 10.6], $\mathrm{p}=0.16$ ) (Fig. 1). However, the median OS of two groups was significantly different; 19.0 months (95\% CI, 14.2 to 23.8) for the WDH group; 14.6 months (95\% CI, 11.9 to 17.3) ( $\mathrm{p}=0.025)$ for the $\mathrm{PDH}$ group (Fig. 2). Again, in numerical perspectives, these data of PDH patients, that is, PFS of 8.9 months and OS of 14.6 months, are better than the data from the typical Asian trial, which might suggest that even patients of PDH could benefit from trastuzumab-based therapy. Other than histologic differentiation, Eastern Cooperative Oncology Group (ECOG) status and serum albumin level were associated with survival (Table 3).

ROC analysis was performed for 59 patients who were confirmed as FISH (+) if a certain HER2/CEP17 ratio could predict favorable PFS for trastuzumab-based treatment. However, we could not determine optimal cutoff values for favorable PFS (Fig. 3) and the AUC was calculated as lower than 0.5 . Higher ratios ( 3 or higher and 4 or higher) were not associated with prolonged PFS (Table 4).

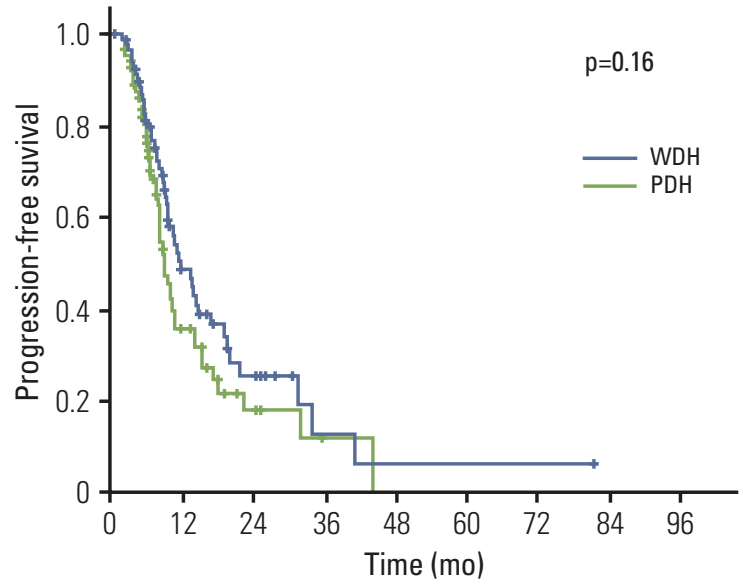

Fig. 1. Progression free survival according to the histologic subtypes; 11.5 months (95\% confidence interval [CI], 8.1 to 14.9) for patients with well-differentiated histology (WDH), and 8.9 months (95\% CI, 7.2 to 10.6) for patients with poorly-differentiated histology (PDH).

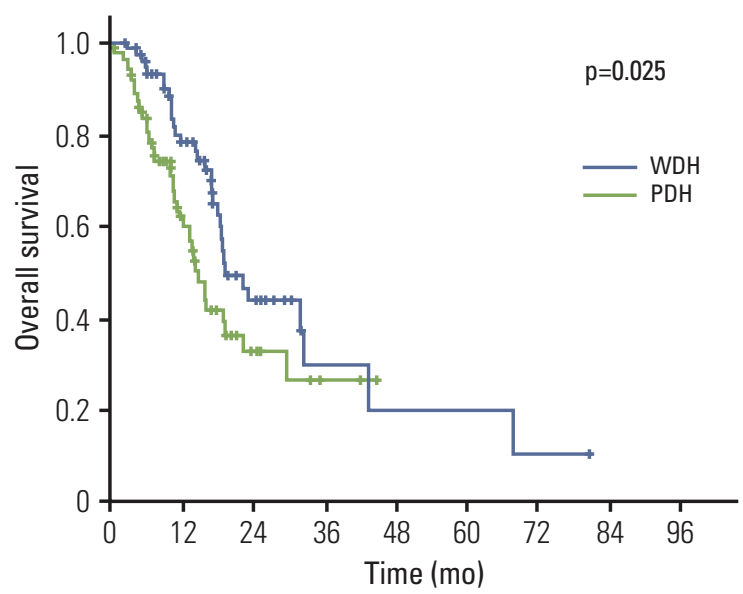

Fig. 2. Overall survival according to the histologic subtypes; 19.0 months (95\% confidence interval [CI], 14.2 to 23.8) for patients with well-differentiated histology (WDH), and 14.6 months (95\% CI, 11.9 to 17.3) for patients with poorly-differentiated histology $(\mathrm{PDH})$. 
Table 3. Survival durations according to other clinicolaboratory factors

\begin{tabular}{|c|c|c|c|c|c|}
\hline Variable & No. & PFS, median $(95 \% \mathrm{CI})$ & p-value & OS, median ( $95 \% \mathrm{CI})$ & p-value \\
\hline \multicolumn{6}{|c|}{ Albumin (g/dL) } \\
\hline$\leq 3.0$ & 19 & $7.3(3.9-10.7)$ & 0.012 & $14.3(7.2-21.4)$ & 0.064 \\
\hline$\geq 3.1$ & 148 & $9.4(5.1-13.7)$ & & $18.7(17.0-20.4)$ & \\
\hline \multicolumn{6}{|l|}{ ECOG PS } \\
\hline 0 & 45 & $14.4(7.8-21.0)$ & 0.005 & $43.4(7.7-79.1)$ & $<0.001$ \\
\hline 1 or 2 & 122 & $9.2(8.7-11.7)$ & & $15.7(13.0-18.4)$ & \\
\hline
\end{tabular}

PFS, progression-free survival; CI, confidence interval; OS, overall survival; ECOG PS, Eastern Cooperative Oncology Group performance status.

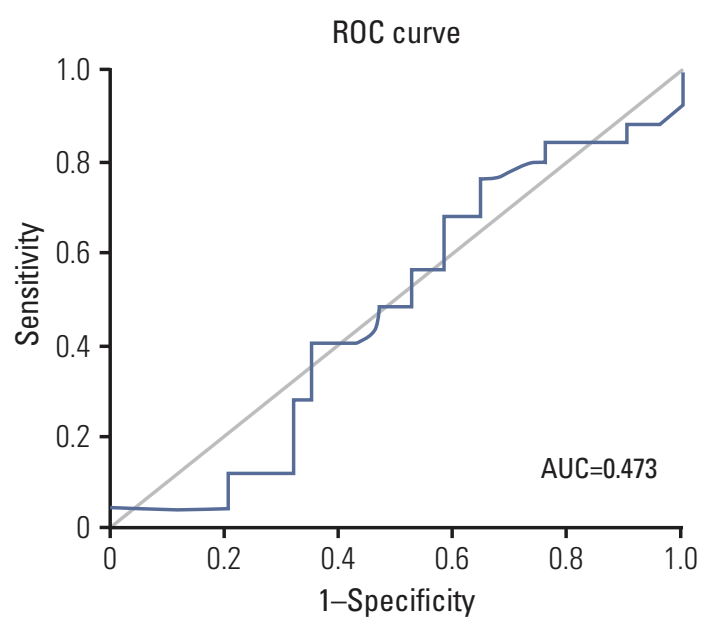

Fig. 3. Receiver operating characteristics (ROC) curves for identification of optimal cutoff values for favorable $(6$ months) progression-free survival. In this analysis, HER2 / CEP17 ratio was not associated with favorable progression-free survival. AUC, area under the curve.

\section{Discussion}

Although several combinations have been introduced in treatment of unresectable or metastatic gastric cancer patients, their prognosis remains poor. As a significant interest in targeted therapies has emerged, a number of targeted agents have been evaluated in gastric cancer. However, most of them fell short of success [7-9], and trastuzumab is the only agent with a known predictive biomarker and proven efficacies. Clinical efficacy of ramucirumab and apatinib was recently demonstrated in refractory gastric cancer patients; however, their predictive biomarkers have not been found.

HER2 is a member of the epidermal growth factor receptor family which promotes cell proliferation and inhibits apop- tosis, and its overexpression has been described in various human malignancies. Trastuzumab, a recombinant humanized anti-HER2 monoclonal antibody, was first developed as a targeted agent for HER2-positive breast cancer, and its unprecedented successes heralded the era of target therapy and relevant biomarkers in the field of oncology. As the carcinogenic roles of HER2 in gastric cancer had been suggested, a randomized phase III trial commenced to assess the clinical efficacy and safety of trastuzumab, which was followed by a great success.

Ethnicity and regional variations should always be considered in management of gastric cancer. Not only the nationwide screening policies, epidemiology, pathogenesis, anatomic location or dominant histology, treatment modalities including surgical approaches and adjuvant treatment also differ between Asian and Western countries [6]. In addition, several pharmacokinetic studies demonstrated that efficacy and tolerability of fluoropyrimidine, a major antineoplastic agent in management of gastric cancer, differ between Asian and Western people [10]. Owing to these factors, the clinical outcomes of Asian gastric cancer patients are superior to those of Western patients. Therefore, mindfulness of ethnicity is important in managing patients and in planning clinical trials as well. Given that HER2 overexpression is frequently found in GEJ and intestinal-type cancer, HER2 overexpression rate might be lower in Asians, who tend to have distal and diffuse-type cancers. However, several series of surveys demonstrated that HER overexpression rate was not low in Asians [11].

As prevalence of gastric cancer is high in Asia, many gastric cancer trials have been conducted with Asians. In the ToGA trial, among 584 patients, 319 (54.6\%) were Asians, and, as described above, Asians per se, were not associated with OS benefit with trastuzumab-based treatment $[5,12]$. However, this could result from the fact that Asian gastric cancer patients already have sufficient survival outcomes with chemotherapy alone, probably due to subsequent treatment which may attenuate the impact of effective combina- 
Table 4. HER2/ CEP17 ratio and progression-free survival $(\mathrm{n}=59)$

\begin{tabular}{lrrc} 
Ratio & No. & PFS, median $(95 \%$ CI) & p-value \\
\hline $0-2.99$ & 22 & $10.2(6.4-14.0)$ & 0.64 \\
$\geq 3$ & 37 & $9.8(5.1-13.7)$ & - \\
$0-3.99$ & 34 & $9.9(6.8-13.0)$ & 0.984 \\
$\geq 4$ & 25 & $9.8(5.7-13.9)$ & - \\
\hline
\end{tabular}

PFS, progression-free survival; CI, confidence interval.

tions as shown in the AVAGAST trial [7]. The benefit of trastuzumab-based therapy in Asians might be suggested in two recent Asian trials. In a Japanese study, 53 patients were treated with trastuzumab combined with S-1 plus cisplatin, and their median PFS and OS were estimated as 7.8 months and 16.0 months [13]. And, in a Korean study, 55 patients treated with trastuzumab combined with capecitabine and oxaliplatin (XELOX) showed a median PFS of 9.8 months and a median OS of 21.0 months [14]. Along with these data, our retrospective analysis showed a median PFS and OS of 10.2 months and 18.5 months, respectively (Table 5). Considering that Asians usually achieve PFS of 5 to 6 months and OS of 12 to 13 months with the first-line fluoropyrimidine and platinum combinations $[7,15]$, these numerical data imply that Asians may also benefit from trastuzumab-based therapy in the same manner with Western people. However, prospective head-to-head comparative analyses are warranted in order to confirm the efficacy of trastuzumab-based therapy in this unique population.

Histologic subtype is one of the interesting issues in HER2positive gastric cancer. Gastric cancer can be categorized according to two distinct histologic subtypes, intestinal and diffuse, which are distinct in their microscopic and gross appearance, epidemiology, pathogenesis, and prognosis [16]. Among them, diffuse-type gastric cancer is associated with poor response to treatment and early relapse, thereby a worse survival [17]. In the current study, we used WHO classification as a substitute for Lauren's classification, as many pathologists in Korea do not interpret Lauren's classification with endoscopic specimens due to fear of misreading, yet a high concordance between two systems has been observed [18]. Compared to the patients with WDH, the patients with PDH showed worse clinical outcomes in both PFS (8.9 months vs. 11.5 months, $\mathrm{p}=0.16$ ) and OS (14.6 months vs. 19.0 months, $\mathrm{p}=0.025)$. As these data are not head-to-head comparisons, it is difficult to make a firm conclusion whether patients with PDH can benefit from trastuzumab-based therapy or not. Nonetheless, considering the numerical clinical data usually reported in Asians, our data may imply that even patients harboring PDH cancer could benefit from trastuzumab-based therapy, although the degree is somewhat limited compared to patients with WDH.

In the perspective of frequency of HER2 overexpression or amplification, it is more commonly found in intestinal-type cancer than diffuse-type cancer. In the ToGA trial, 51 out of 584 patients $(8.7 \%)$ had diffuse-type cancer, and in the LOGiC trial, the differential became even more remarkable; $3.9 \%$ of patients $(19 / 487)$ had diffuse-type cancer [19]. This dominance of intestinal-type cancer in the two trials looks suitable considering its disease nature. However, in our cohort, the number of patients with PDH $(n=86,51.2 \%)$ was slightly higher than that of patients with $\mathrm{WDH}(\mathrm{n}=77,45.8 \%)$. This could have resulted from the histologic distribution of gastric cancer patients in Asia. Several large studies from

Table 5. Comparison of clinical outcomes of anti-HER2 trials for Asians

\begin{tabular}{|c|c|c|c|c|c|}
\hline Trial & Regimen & ORR $(\%)$ & PFS, median (95\% CI, mo) & OS, median (95\% CI, mo) & No. \\
\hline HERBIS-1 [13] & $\begin{array}{l}\text { Trastuzumab }(8 \rightarrow 6 \mathrm{mg} / \mathrm{kg}) \\
\text { SP }\end{array}$ & 68 & $7.8(6.0-8.8)$ & $16.0(13.3-\mathrm{N} / \mathrm{A})$ & 53 \\
\hline Ryu et al. [14] & $\begin{array}{l}\text { Trastuzumab }(8 \rightarrow 6 \mathrm{mg} / \mathrm{kg}) \\
\text { XELOX }\end{array}$ & 68 & $9.8(7.0-12.6)$ & $21.0(6.4-35.7)$ & 55 \\
\hline Present study & $\begin{array}{l}\text { Trastuzumab }(8 \rightarrow 6 \mathrm{mg} / \mathrm{kg}) \\
\text { Various backbone regimens }\end{array}$ & 50.6 & $10.2(8.7-11.7)$ & $18.5(16.4-50.6)$ & 168 \\
\hline ToGA trial [12] $]^{\text {a) }}$ & $\begin{array}{l}\text { Trastuzumab }(8 \rightarrow 6 \mathrm{mg} / \mathrm{kg}) \\
\text { XP or FP }\end{array}$ & 64.4 & $6.2(5-7)$ & $15.9(12-25)$ & 51 \\
\hline LOGiC trial [19] $]^{\mathrm{b})}$ & $\begin{array}{l}\text { Lapatinib } 1,250 \mathrm{mg} \text { every day } \\
\text { XELOX }\end{array}$ & $\mathrm{N} / \mathrm{A}$ & $\mathrm{N} / \mathrm{A}$ & $16.5(13.3-20.2)$ & 100 \\
\hline
\end{tabular}

ORR, objective response rate; PFS, progression-free survival; CI, confidence interval; OS, overall survival; SP, S-1 plus cisplatin; XELOX, capecitabine and oxaliplatin; XP, capecitabine plus cisplatin; FP, 5-fluorouracil plus cisplatin; N/ A, not avail-

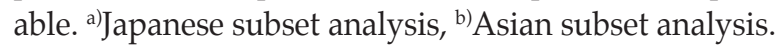


Asia demonstrated that diffuse-type or poorly-differentiated cancer was more predominant $[15,20]$. Although these types of gastric cancer do not harbor HER2 overexpression very often, out-numbering of patients could draw the results. Similar to our finding, in the TyTAN trial, which recruited HER2-positive gastric cancer patients confined to Asia [21], only $43 \%$ of patients had intestinal-type gastric cancer.

A HER2/CEP17 ratio was recently suggested as a novel predictive marker for trastuzumab-based treatment. In a Spanish trial, 66 FISH positive patients were evaluated and HER2/CEP17 higher than 4.7 was identified as the cutoff value for prolonged OS [22]. In another report by Ock et al. [23], a HER2/CEP17 ratio of 4.48 clearly discriminated clinical outcomes. In our study, however, we failed to find optimal discriminating values for favorable PFS. This could have resulted from the small number of samples, and our FISH data did not come from a single laboratory. Tumor heterogeneity regarding the HER2 amplification could also be another factor [24]. Further analysis is requested on this issue.

The current study has several limitations. Data were collected in a retrospective manner and from multiple institutes. This could draw somewhat inconsistent interpretation regarding the favorable tumor response, such as complete or partial responses. However, as we assessed PFS and OS, we believe the validity of the conclusive data and rather, it may reflect real world clinical outcomes. Although the backbone regimens are varied, most of them $(98.2 \%)$ are the combination of fluoropyrimidine (5-FU and capecitabine) and platinum (cisplatin, oxaliplatin) which have similar efficacies and can be used interchangeably. And, as we focused on the efficacy of the treatment, the current analysis does not provide ethnic or histologic difference of safety issue.

\section{Conclusion}

In conclusion, despite the result of subset analysis of the ToGA trial, we might conclude that irrespective of ethnicity or histologic subtypes, patients with HER2-positive gastric cancer can benefit from trastuzumab-based chemotherapy. Further analysis to find predictive biomarkers for these patients should be continued and ethnic difference and histologic subtypes should be considered for future clinical trials on gastric cancer patients.

\section{Conflicts of Interest}

Conflict of interest relevant to this article was not reported.

\section{Author Details}

${ }^{1}$ Division of Hematology-Oncology, Department of Internal Medicine, Hanyang University Seoul Hospital, Seoul, ${ }^{2}$ Division of Hematology-Oncology, Department of Internal Medicine, Gyeongsang National University School of Medicine, Jinju, ${ }^{3}$ Division of Hematology/Oncology, Department of Internal Medicine, Chung-Ang University Hospital, ChungAng University College of Medicine, Seoul, ${ }^{4}$ Division of Hematology/Oncology, Department of Internal Medicine, Gachon University Gil Hospital, Incheon, ${ }^{5}$ Department of Internal Medicine, Dankook University College of Medicine, Cheonan, ${ }^{6}$ Division of Hematology and Oncology, Department of Internal Medicine, Hanyang University Guri Hospital, Hanyang University College of Medicine, Guri, 'Division of Hematology and Oncology, Department of Medicine, Samsung Changwon Hospital, Sungkyunkwan University, Changwon, ${ }^{8}$ Division of Oncology, Department of Medicine, Yonsei University, Severance Hospital, Seoul, ${ }^{9}$ Department of Internal Medicine, Dong-A University College of Medicine, Busan, ${ }^{10}$ Department of Internal Medicine, Ewha Womans University School of Medicine, Seoul, ${ }^{11}$ Division of Hematology-Oncology, Department of Internal Medicine, VHS Medical Center, Seoul, ${ }^{12}$ Division of HematologyOncology, Department of Internal Medicine, Kyung Hee University School of Medicine, Seoul, ${ }^{13}$ Division of Hematology-Oncology, Department of Medicine, Samsung Medical Center, Sungkyunkwan University School of Medicine, Seoul, Korea 


\section{References}

1. Ferlay J, Soerjomataram I, Dikshit R, Eser S, Mathers C, Rebelo $\mathrm{M}$, et al. Cancer incidence and mortality worldwide: sources, methods and major patterns in GLOBOCAN 2012. Int J Cancer. 2015;136:E359-86.

2. Jemal A, Bray F, Center MM, Ferlay J, Ward E, Forman D. Global cancer statistics. CA Cancer J Clin. 2011;61:69-90.

3. Al-Batran SE, Hartmann JT, Probst S, Schmalenberg H, Hollerbach S, Hofheinz R, et al. Phase III trial in metastatic gastroesophageal adenocarcinoma with fluorouracil, leucovorin plus either oxaliplatin or cisplatin: a study of the Arbeitsgemeinschaft Internistische Onkologie. J Clin Oncol. 2008;26:1435-42.

4. Van Cutsem E, Moiseyenko VM, Tjulandin S, Majlis A, Constenla M, Boni C, et al. Phase III study of docetaxel and cisplatin plus fluorouracil compared with cisplatin and fluorouracil as first-line therapy for advanced gastric cancer: a report of the V325 Study Group. J Clin Oncol. 2006;24: 4991-7.

5. Bang YJ, Van Cutsem E, Feyereislova A, Chung HC, Shen L, Sawaki A, et al. Trastuzumab in combination with chemotherapy versus chemotherapy alone for treatment of HER2-positive advanced gastric or gastro-oesophageal junction cancer (ToGA): a phase 3, open-label, randomised controlled trial. Lancet. 2010;376:687-97.

6. Macdonald JS. Gastric cancer: Nagoya is not New York. J Clin Oncol. 2011;29:4348-50.

7. Ohtsu A, Shah MA, Van Cutsem E, Rha SY, Sawaki A, Park $\mathrm{SR}$, et al. Bevacizumab in combination with chemotherapy as first-line therapy in advanced gastric cancer: a randomized, double-blind, placebo-controlled phase III study. J Clin Oncol. 2011;29:3968-76.

8. Lordick F, Kang YK, Chung HC, Salman P, Oh SC, Bodoky G, et al. Capecitabine and cisplatin with or without cetuximab for patients with previously untreated advanced gastric cancer (EXPAND): a randomised, open-label phase 3 trial. Lancet Oncol. 2013;14:490-9.

9. Ohtsu A, Ajani JA, Bai YX, Bang YJ, Chung HC, Pan HM, et al. Everolimus for previously treated advanced gastric cancer: results of the randomized, double-blind, phase III GRANITE1 study. J Clin Oncol. 2013;31:3935-43.

10. Ajani JA, Faust J, Ikeda K, Yao JC, Anbe H, Carr KL, et al. Phase I pharmacokinetic study of S-1 plus cisplatin in patients with advanced gastric carcinoma. J Clin Oncol. 2005;23: 6957-65.

11. Kim KM, Bilous M, Chu KM, Kim BS, Kim WH, Park YS, et al. Human epidermal growth factor receptor 2 testing in gastric cancer: recommendations of an Asia-Pacific task force. Asia Pac J Clin Oncol. 2014;10:297-307.

12. Sawaki A, Ohashi Y, Omuro Y, Satoh T, Hamamoto Y, Boku $\mathrm{N}$, et al. Efficacy of trastuzumab in Japanese patients with HER2-positive advanced gastric or gastroesophageal junction cancer: a subgroup analysis of the Trastuzumab for Gastric Cancer (ToGA) study. Gastric Cancer. 2012;15:313-22.
13. Kurokawa Y, Sugimoto N, Miwa H, Tsuda M, Nishina S, Okuda $\mathrm{H}$, et al. Phase II study of trastuzumab in combination with S-1 plus cisplatin in HER2-positive gastric cancer (HERBIS-1). Br J Cancer. 2014;110:1163-8.

14. Ryu MH, Yoo C, Kim JG, Ryoo BY, Park YS, Park SR, et al. Multicenter phase II study of trastuzumab in combination with capecitabine and oxaliplatin for advanced gastric cancer. Eur J Cancer. 2015;51:482-8.

15. Koizumi W, Narahara H, Hara T, Takagane A, Akiya T, Takagi $\mathrm{M}$, et al. S-1 plus cisplatin versus S-1 alone for first-line treatment of advanced gastric cancer (SPIRITS trial): a phase III trial. Lancet Oncol. 2008;9:215-21.

16. Lauren P. The two histological main types of gastric carcinoma: diffuse and so-called intestinal-type carcinoma. An attempt at a histo-clinical classification. Acta Pathol Microbiol Scand. 1965;64:31-49.

17. Smalley SR, Benedetti JK, Haller DG, Hundahl SA, Estes NC, Ajani JA, et al. Updated analysis of SWOG-directed intergroup study 0116: a phase III trial of adjuvant radiochemotherapy versus observation after curative gastric cancer resection. J Clin Oncol. 2012;30:2327-33.

18. Qiu M, Zhou Y, Zhang X, Wang Z, Wang F, Shao J, et al. Lauren classification combined with HER2 status is a better prognostic factor in Chinese gastric cancer patients. BMC Cancer. 2014;14:823.

19. Hecht JR, Bang YJ, Qin S, Chung HC, Xu JM, Park JO, et al. Lapatinib in combination with capecitabine plus oxaliplatin (CapeOx) in HER2-positive advanced or metastatic gastric, esophageal, or gastroesophageal adenocarcinoma (AC): The TRIO-013/LOGiC Trial. J Clin Oncol. 2013;31 Suppl:Abstr LBA4001.

20. Sakuramoto S, Sasako M, Yamaguchi T, Kinoshita T, Fujii M, Nashimoto A, et al. Adjuvant chemotherapy for gastric cancer with S-1, an oral fluoropyrimidine. N Engl J Med. 2007;357: 1810-20.

21. Satoh T, Xu RH, Chung HC, Sun GP, Doi T, Xu JM, et al. Lapatinib plus paclitaxel versus paclitaxel alone in the second-line treatment of HER2-amplified advanced gastric cancer in Asian populations: TyTAN: a randomized, phase III study. J Clin Oncol. 2014;32:2039-49.

22. Gomez-Martin C, Plaza JC, Pazo-Cid R, Salud A, Pons F, Fonseca $\mathrm{P}$, et al. Level of HER2 gene amplification predicts response and overall survival in HER2-positive advanced gastric cancer treated with trastuzumab. J Clin Oncol. 2013;31: 4445-52.

23. Ock CY, Lee KW, Kim JW, Kim JS, Kim TY, Lee KH, et al. Optimal patient selection for trastuzumab treatment in HER2positive advanced gastric cancer. Clin Cancer Res. 2015;21: 2520-9.

24. Lee HE, Park KU, Yoo SB, Nam SK, Park DJ, Kim HH, et al. Clinical significance of intratumoral HER2 heterogeneity in gastric cancer. Eur J Cancer. 2013;49:1448-57. 